\title{
Nanoexposure, Unusual Diseases, and New Health and Safety Concerns
}

\author{
Yuguo Song ${ }^{1}$ and Shichuan Tang ${ }^{2}$ \\ ${ }^{1}$ Department of Occupational Medicine and Clinical Toxicology, \\ Beijing Chaoyang Hospital, Capital University of Medical Sciences, \\ No. 8 Gongtinan Road, Chaoyang District, Beijing 100020, China \\ ${ }^{2}$ Beijing Key Laboratory of Occupational Health and Safety, \\ Beijing Municipal Institute of Labor Protection, Beijing 100054, China
}

Received 28 April 2011; Accepted 13 September 2011

Academic Editor: Denis Girard

Accumulating studies in animals have shown that nanoparticles could cause unusual rapid lung injury and extrapulmonary toxicity. Whether exposure of workers to nanoparticles may result in some unexpected damage as seen in animals is still a big concern. We previously reported findings regarding a group of patients exposed to nanoparticles and presenting with an unusual disease. The reported disease was characterized by bilateral chest fluid, pulmonary fibrosis, pleural granuloma, and multiorgan damage and was highly associated with the nanoparticle exposure. To strengthen this association, further information on exposure and the disease was collected and discussed. Our studies show that some kinds of nanomaterials, such as silica nanoparticles and nanosilicates, may be very toxic and even fatal to occupational workers exposed to them without any effective personal protective equipment. More research and collaborative efforts on nanosafety are required in order to prevent and minimize the potential hazards of nanomaterials to humans and the environment.

KEYWORDS: silica nanoparticle, nanosilicate, lung injury, pleural effusion 


\section{INTRODUCTION}

With the development and wide use of nanomaterials, concerns and fears have been expressed regarding potential risks of nanoparticles to worker's safety, human health, and environment contamination [1]. Due to their unique physicochemical properties such as extremely small size, large surface area, and functional coating, nanomaterials may exhibit nonclassic bioactivity and interact with cellular or subcellular structures. And accumulating research data using animal or cultured cell models have shown nanoparticles could cause unusual rapid lung injury and extrapulmonary toxicity $[2,3]$. It is possible theoretically that long-term exposure to nanoparticles may cause some unexpected damage to humans as seen in animals.

We previously reported findings regarding a group of patients, who worked at the same workplace in a print plant for 5-13 months exposing to nanoparticles [4]. These workers presented with dyspnea on exertion pericardial and pleural effusions and rash with intense itching. The symptoms seen in these patients were similar to those seen in animals exposed to nanoparticles. In addition, considering particle size and deposition model, the damage in lungs was highly associated with the nanoparticle exposure. To strength this association, further information on exposure and disease was collected. In this paper, we will discuss these unusual diseases and their nanoexposure.

\section{UNUSUAL DISEASE AND NANOEXPOSURE}

Our seven patients, 18-47 years old female workers, were all previously in good health and nonsmokers. They were found in almost the same time frame with the same symptoms of shortness of breath and the same clinical findings such as pleural effusion, progressive pulmonary fibrosis, and pleural damage. In addition, multiorgan damage was observed including disorders of haematotoxicity, cardiotoxicity, hepatotoxicity, and dermal toxicity. And a two-year follow-up revealed that two patients died of pulmonary failure and five were disabled with severe pulmonary dysfunction.

In the early disease stage $[4,5]$, the pathological examination showed the effusion of inflammatory cells in tunica mucosa bronchiorum, aggregations of macrophages and inflammatory cells, proteinaceous effusions in the alveolar space, swollen and widened alveolar septums with scattered neutrophil leukocytes, and pulmonary fibrosis. And pathological study of pleura exhibited fibrinous and inflammatory cells, foreign-body granulomas, and haemorrhage. In the late stage, damage to pulmonary tissue was similar to but more prominent than that in the early stage. Pulmonary alveoli were partly emphysematous with aggregations of macrophages and type II alveolar epithelial cell proliferations, and the alveolar septum was widened with blood vessel dilatation and congestion. Notably, damage to alveolar epithelial cells, macrophages, vascular endothelial cells, and the blood-gas barrier was also observed.

All the patients are characterized with pleural effusions. Some common causes of pleural effusions are lung infections, tuberculosis, pulmonary embolism, breast cancer, lung cancer, or autoimmune diseases. However, clinical examinations and long-term follow-up have eliminated the possibility of above diseases in our patients.

\section{EXPOSURE IN THE WORKPLACE}

The coating material used in the workplace, an ivory white soft paste (polyacrylic ester stated by the paste producer), was analyzed by gas chromatography/mass spectrometry (GC/MS), and was found containing the following components [4]: butanoic acid, butyl ester, N-butyl ether, acetic acid, toluene, di-tert-butyl peroxide, 1-butanol, acetic acid ethenyl ester, isopropyl alcohol, and ethylene dioxide. Unfortunately, these patients exhibited none of the signs and symptoms related to these irritant or asphyxiant gases, such as coughing, eye irritation, dizziness/headaches, drowsiness, nausea, or vomiting. In addition, the powder component in the raw materials used in the workplace was determined by inductively coupled plasma atomic 
emission spectrometry (ICP-AES) and was found containing (\%): Si 0.016, Ba 0.069, Ca 0.20, Mg 0.051, $\mathrm{Cu} 0.0090, \mathrm{Fe} 0.098, \mathrm{~K} 0.015, \mathrm{Na} 0.82$, Zn 0.0085, and P $0.048[5,6]$.

Nanoparticles found in the patients' pulmonary tissues and chest effusions were the same raw materials used by these workers. Element of silica of nanoparticles was identified in biopsies and chest fluid by using transmission electron microscopy and energy dispersive X-ray analysis. They were largely found in macrophages (cytoplasm, mitochondria, lysosomes, and nuclei), pulmonary microvascular vessels, vascular endothelial cells, microlymphatic vessels, and pleural effusions. We also observed silica nanoparticles accumulated in alveolar epithelial cells and pulmonary interstitial tissue but to a less extent $[4,5]$. In addition, nanosilicates, as well as microscale silicates, were also observed in a patient pleural effusion and contained elements of $\mathrm{Si}, \mathrm{Ca}, \mathrm{Mg}, \mathrm{K}, \mathrm{Na}, \mathrm{Ba}, \mathrm{Al}, \mathrm{P}, \mathrm{S}, \mathrm{Cl}$, and $\mathrm{O}$ [5].

The concordance of the components of powder in the raw material and nanomaterials in patients' biopsies indicated that the powder in the raw material was made of silica nanoparticles and silicates, a mixture used to obtain some special coating quality, such as abrasive resistant, flame retardant, or adherent. Thus, a rough sketch can be made of the coatings used at these patients' workplace: filters and additives of silica nanoparticles and nano- and microscale silicates, a film-forming polyacrylate agent, and various solvents, including butanoic acid, butyl ester, acetic acid, and toluene.

\section{WHY WERE SILICA NANOPARTICLS AND NANOSILICATES USED TOGETHER IN THE PRODUCTION OF NANOCOATINGS AND FOUND IN THE COATINGS USED IN THE WORKPLACE?}

Coatings usually have several components of binders, pigments, solvents, fillers, and additives [7]. Fillers and additives are generally composed of natural or synthetic minerals, such as silicon dioxide, barium sulfate, silicates, or a complex mixture of the above materials, to produce special and improved functions, such as water resistance, transparency, and flame retardation [7,8]. Due to their small size, large specific surface area, and more alkyl groups in different bonding states, silica nanoparticles in coatings can markedly improve the suspension stability, weatherability, tensile strength, and the washing, infrared, abrasion, heat, and radiation resistances of a coating and even yield the coating clear at the same time [8,9]. However, microsilica can only increase the hardness and abrasion resistance [9]. Similarly, Layered silicates, another very popular coating nanomaterial ingredient, play an important role as adsorbents, catalysts, and ionic exchangers in the production of nanocoatings and could greatly increase thermal stability and act as fire retardants in the form of nanocomposites [10].

Nanosilicates, often characterized by only one dimension in the nanometer range, are a group of very complex chemical substances in which there is a copolymerization of silicic acid with hydroxides of other metals drawn from almost the entire periodic table [11], while silicates isolated in our patients contain 13-15 different elements and widely varying $\mathrm{O} / \mathrm{Si}$ ratios. Their structure consists of layers made up of two tetrahedrally coordinated silicon atoms fused to an edge-shared octahedral sheet of either aluminum or magnesium hydroxide. Using layered nanosilicates in nanomaterials, several structural types may be obtained, including intercalated nanocomposites in which polymer chains are sandwiched between silicate layers and exfoliated nanocomposites in which separated, individual, silicate layers are more or less uniformly dispersed in a polymer matrix. These nanocomposites exhibit markedly improved mechanical, thermal, optical, and physicochemical properties compared with pure polymers or conventional (microscale) composites [10].

Given the benefits of silica nanoparticles and nanosilicates, they are usually utilized concurrently in varying ratios to each other in the production of nanocomposite coatings. And this may be the very reason that amorphous silica and silicates in nanoscale were found in the raw materials that these workers exposed to. 


\section{EXPOSURE TO SILICA AND/OR SILICATE RESULTS IN PNEUMOCONIOSIS, WHAT IF EXPOSURE TO SILICA AND/OR SILICATE IN NANOSCALE?}

Occupational exposures to inhaled pathogenic particles and fibers such as silica and silicate have been associated with the development of lung inflammation and occupational disease. These diseases include silicosis, interstitial fibrosis, industrial bronchitis, small airway disease, emphysema, and vascular diseases as well as immunologic reactions $[12,13]$. Even though amorphous silica appears less toxic than the crystalline form, toxicological studies about amorphous silica nanoparticles have exhibited that they increase reactive oxygen species (ROS) concentrations, reduce glutathione levels, and induce pro-inflammatory, inflammatory, and oxidative stress responses both in vivo and in vitro $[14,15]$. Amorphous silica nanoparticles can enter cell nuclei where reducing aberrant clusters of topoisomerase I and protein aggregates in the nucleoplasm [16]. In vitro studies also show that amorphous silica nanoparticles disturb mitochondrial function and lower cell survival via decreasing cell survival signaling in human neural cells [17]. Furthermore, amorphous nanosilica has been shown to accumulate in the lung, liver, kidney, gut, bone marrow, and brain in animal experiments and cause multiorgan damage [14].

Exposure to silica and/or silicate results in pneumoconiosis, what if exposure to silica and/or silicate in nanoscale? Given the well-documented toxicity of microscale silica in humans and findings of amorphous silica nanoparticles in animals, it is possible that these silica nanoparticles may have been contributed in part to these patients' disease and multiorgan damage, whatever they exerted toxicities directly or acted as carriers. These patients were found multiorgan damage, which may have been related to nanoparticle entry into the systematic circulation and multiorgan deposition. These silica nanoparticles in the nuclei may potentially bind to the DNA phosphate backbone, influence nuclear integrity, and trigger genotoxicity by physical-chemical interaction, forming intranuclear protein aggregates, and regulating redoxsensitive transcription factors and DNA-damage responsive signaling, as seen in animal-based nanotoxicological studies [16-18], and also, these silica nanoparticles in cytoplasm may exert cytotoxicity by increasing reactive oxygen species and by inducing proinflammatory, inflammatory, and oxidative stress responses, as seen both in vivo and in vitro $[14,15]$.

\section{THE POTENTIAL TRANSLOCATION PATHWAYS OF NANOPARTICLES AND THEIR POTENTIAL DAMAGE}

A hypothesis of the potential translocation pathways of nanoparticles and their potential damage was made as follows (Figure 1). When coatings were sprayed, heated, and dried in the workplace, nanosilica and nanosilicates in these coatings aired in the workplace as flocculi (condensation of the aerosol, containing nanosilica and nanosilicates from the volatilized material) and caused itching on the faces and arms of patients (dermal toxicity), while the workers used no personal protective equipment (PPE), because the nanomaterials were invisible and they knew nothing of the toxicity of the materials they were handling. When inhaled into the pulmonary respiratory tract, the nanomaterials deposited in the pulmonary alveoli were taken up by macrophages, crossed the lung epithelium, and entered the pulmonary interstitial tissue, blood, and lymph, finally reaching extrapulmonary organs of patients, as has been observed in animal experiments [14], and resulted in the haematotoxicity, hepatotoxicity, cardiotoxicity, and pulmonary and pleural injuries seen in these patients. The nanomaterials within pleural effusions may have come from visceral pleura through the pulmonary circulation and from partial pleura through the systemic circulation, or come from penetration through lung tissue to the pleural membrane, as small-sized nanomaterials like nanosilica can readily pass through lung tissue or behave like gases [19]. Finally, chronic and repetitive exposure (5-13 months) to the nanomaterials may have resulted in these patients' illness and death.

In our patients, the initial and persistent symptoms were the recurring production of copious amounts of chest fluids. The likelihood of patients developing pleural effusion is a combined effect of nanosilicates and nanosilica, which can be obtained as an indication from the pleural effusions caused by silicosis or asbestosis [20]. The occurrence of pleural effusion may have been caused not by the chemical compositions 


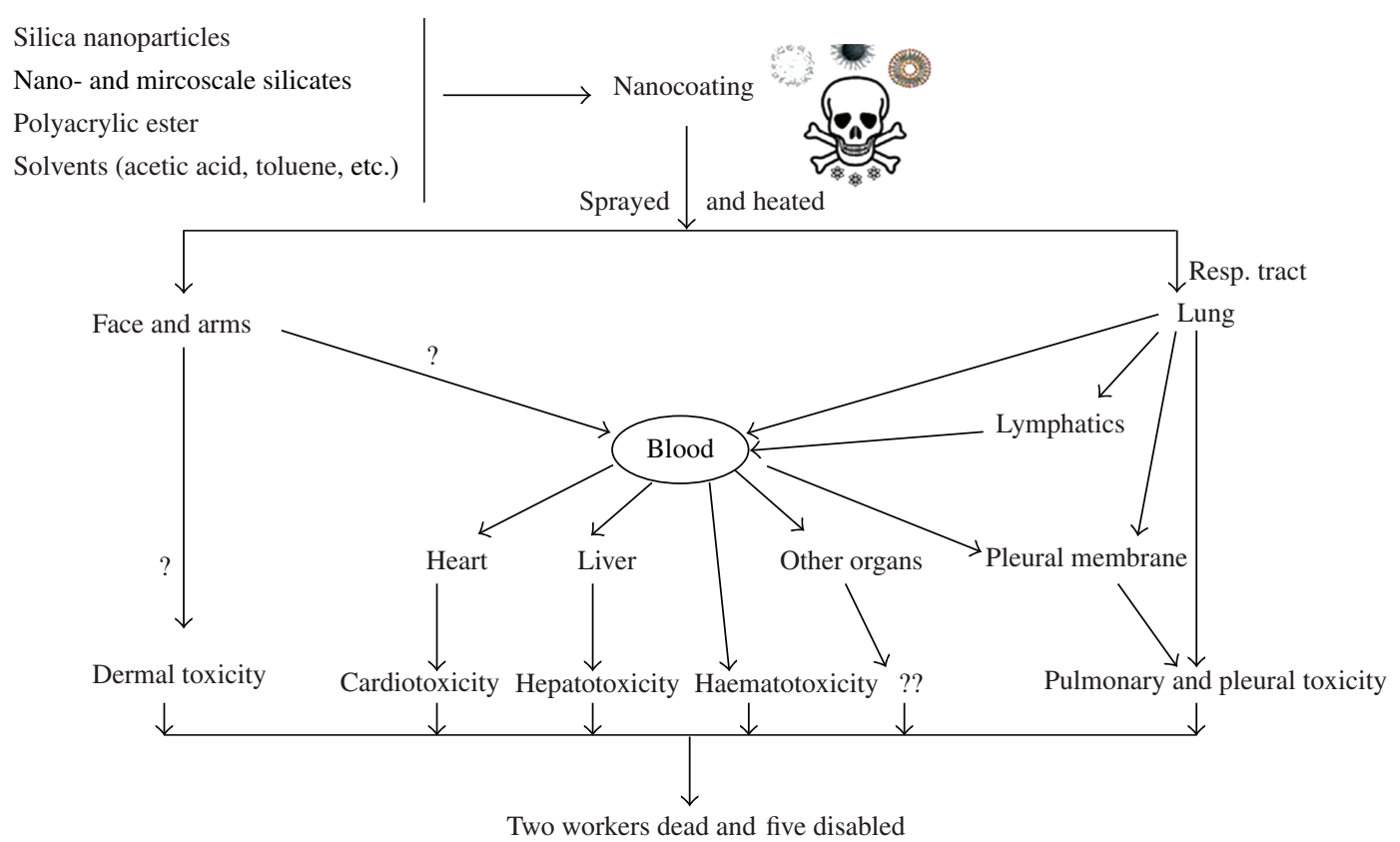

FIGURE 1: Potential translocation pathways of nanoparticles and the potential damage. Nanosilica and nanosilicates in the coatings may air in the workplace when sprayed, heated, and dried, then they deposit in the pulmonary alveoli when inhaled into the pulmonary respiratory tract, be taken up by macrophages, cross the lung epithelium, enter the pulmonary interstitial tissue, blood, and lymph, finally reach extrapulmonary organs, and result in multiorgan toxicities.

of the nanomaterials but by their nanosize. These nanomaterials are small enough to pass through the lung tissue into the pleural cavity but large enough to block proper lymphatic drainage of the pleural membrane. Furthermore, lymphatic flow under physiological conditions amounts to $3 \mu \mathrm{L} / \mathrm{min} / \mathrm{mm}^{2}$ and flow velocity in initial lymphatics is on the order of $2 \mathrm{~mm} / \mathrm{min}$ [21], which is much slower than blood flow velocity and thus prone to pleural lymphatic stomata blocking. Importantly, the draining role of parietal lymphatics in patients with asbestosis is demonstrated by the finding of the "black spots," the accumulation of asbestos fibers around the parietal pleural lymphatic stomata due to the fibers being flow-driven towards the pleural fluid draining sites $[22,23]$. Also, the presumption that pleural effusions in our patients may have been caused by nanomaterials blocking proper lymphatic drainage of the pleural cavity was confirmed by the observation of lymphangiectasis and lymphostasis in high-resolution CT scans of lungs and pathologic observation of lungs and pleural membranes.

\section{NEW HEALTH AND SAFETY CONCERNS}

Our findings of nanosilica in patients' biopsies and pleural effusions, together with our previous report, present a clue that these silica nanoparticles may have contributed in part to the illness reported in these workers. The reported disease was characterized by bilateral chest fluid, pulmonary fibrosis, and pleural damage and was featured by a concealed onset (a relatively long latency between initial exposure and disease emergence), rapid and progressive development, and multiorgan damage. These characteristics varied greatly from diseases such as silicosis or asbestosis which were caused by microscale silica or silicates. Thus, the disease related to nanomaterials produces new challenges and concerns in terms of how to prevent the potential risks of nanomaterial exposure to workers and the environment as nanomaterials are invisible. 
(1) This study highlights the urgent need for safety practice guideline to protect workers. Protocols or solutions may include regulations for occupational health and safety (OHS) in nanofield work or research, medical prescreening and surveillance, establishing industrial hygiene guidelines, and effective PPE or application of appropriate safeguards. In reality, conventional OHS practices when handling nanomaterials are usually employed, but the effectiveness of the application of these classical tools is in doubt. For example, a traditional cotton mask usually has apertures $100-300 \mu \mathrm{m}$ in diameter, much larger than nanoscale, implying that using a cotton mask to avoid nanomaterial exposure would be ineffective.

(2) A complete assessment of the potential risks and impacts of nanomaterials and nanoproducts on human health and the environment is essential before they enter commercial markets. Over 1900 different types of nanomaterials have been designed and fabricated, each of which having distinct physicochemical features, but only dozens of these materials have been studied so far, and there is little data regarding human toxicity. Additionally, novel, engineered nanomaterials will increasingly be produced to maximize beneficial physicochemical features, and, concurrently, more and more nanomaterials will be discarded at the end of their useful life. Hence, human exposure is already occurring and will increase dramatically in coming years. Full assessment of the potential risks of nanomaterials is essential to avoid future horrible scenarios as studied here. Nanomaterials should be considered hazardous until proven otherwise.

(3) Nanomaterials in products should be clearly stated or labeled. On the one hand, nanomaterial manufacturers do not claim what is in their products. On the other hand, the merchants use the concept of "nano" for marketing. Actually, in our opinion, the injury or suspected injury related to nanomaterials are observed among consumers than workers, as consumers are not aware of the risk of the products they are exposed to.

(4) Given the severity of disease related to some nanomaterials, such as nanosilica and nanosilicates and the great difficulties in diagnosis and treatment, it is a new challenge for our clinical doctors. Much more work including clinical laboratory studies of nanotoxicology is required to prevent, diagnose, and treat disease or injury related to nanomaterials.

(5) A multidisciplinary collaborative approach, involving at least physicians, material scientists, epidemiologists, and toxicologists, should be followed to probe into the association and detailed mechanisms of nanomaterials with cardiovascular disease, respiratory disease (e.g., asthma, chronic inflammation, and cancer), and mortality as well as immunologic diseases.

(6) Supervision of the usage of nanomaterials, which are easily available, invisible, and possibly highly toxic, as seen in our patients, should be imposed to prevent the occurrence of a similar accidental disaster and also to prevent the potential use of these materials as new weapons by criminals and terrorists.

(7) More research and collaborative efforts, especially on particular materials, such as the nanosilica and nanosilicates found in our patients' biopsies, are required to gain a broad and specific understanding of the distribution, biokinetics, and toxicity of these materials in the human body. Also, more government funding and private investment in nanotoxicology are essential to provide the resources to allow extensive understanding of the effects of nanomaterials and nanowaste on humans and the environment.

(8) Ecotoxicological study to assess the potential entry, hazard, and risk of nanomaterials to the ecosystem for their safer use and handling has accordingly been the topic of great interest and urgency.

In conclusion, our studies show that some kinds of nanomaterials, such as silica nanoparticles and nanosilicates, may be very toxic and even fatal to occupational workers exposed to but without any effective PPE. And the disease related to these nanomaterials produces new health and safety concerns on how to prevent the potential risks of nanomaterial exposure to workers and the environment as well as how to clinically 
diagnose and treat the disease or injury. More research and collaborative efforts on nanosafety, and more government funding and private investment in nanotoxicology are required in order to prevent and minimize the potential hazards of nanomaterials to humans and the environment.

\section{ACKNOWLEDGMENTS}

The author sincerely thanks all the doctors and nurses for their hard work in the treatment of these patients. This work was supported in part by National Natural Science Foundation of China (Grant no. 81172614), Knowledge Innovation Project of Beijing Academy Science and Technology (PXM2011-178304-112365) and Beijing New Century Talents Projects (2009BJRS001).

\section{REFERENCES}

[1] A. Nel, T. Xia, L. Madler, and N. Li, “Toxic potential of materials at the nanolevel," Science, vol. 311, no. 5761, pp. 622-627, 2006.

[2] R. R. Mercer, J. Scabilloni, L. Wang et al., "Alteration of deposition pattern and pulmonary response as a result of improved dispersion of aspirated single-walled carbon nanotubes in a mouse model," American Journal of Physiology_Lung Cellular and Molecular Physiology, vol. 294, no. 1, pp. L87-L97, 2008.

[3] D. B. Warheit, B. R. Laurence, K. L. Reed, D. H. Roach, G. A. M. Reynolds, and T. R. Webb, "Comparative pulmonary toxicity assessment of single-wall carbon nanotubes in rats," Toxicological Sciences, vol. 77, no. 1, pp. 117-125, 2004.

[4] Y. Song, X. Li, and X. Du, "Exposure to nanoparticles is related to pleural effusion, pulmonary fibrosis and granuloma," European Respiratory Journal, vol. 34, no. 3, pp. 559-567, 2009.

[5] Y. Song, X. Li, L. Wang et al., "Nanomaterials in humans: identification, characteristics, and potential damage," Toxicologic Pathology, vol. 39, no. 5, pp. 841-849, 2011.

[6] Z. F. Ma, A virulence study for organic polymer A causing lungs tissue damage in rats, M.S. thesis, Hebei United University, Tangshan, China, 2009.

[7] G. J. Liu, Nanomaterials and Modified Coatings, Chemical Industry Press (CIP), Beijng, China, 1 st edition, 2008.

[8] Z. Wang, E. Han, and W. Ke, "An investigation into fire protection and water resistance of intumescent nanocoatings," Surface and Coatings Technology, vol. 201, no. 3-4, pp. 1528-1535, 2006.

[9] S. Zhou, L. Wu, J. Sun, and W. Shen, "The change of the properties of acrylic-based polyurethane via addition of nano-silica," Progress in Organic Coatings, vol. 45, no. 1, pp. 33-42, 2002.

[10] D. F. Schmidt and E. P. Giannelis, "Silicate dispersion and mechanical reinforcement in polysiloxane/layered silicate nanocomposites," Chemistry of Materials, vol. 22, no. 1, pp. 167-174, 2010.

[11] R. Y. Ning, "Discussion of silica speciation, fouling, control and maximum reduction," Desalination, vol. 151, no. 1, pp. 67-73, 2003.

[12] M. Ding, F. Chen, X. Shi, B. Yucesoy, B. Mossman, and V. Vallyathan, "Diseases caused by silica: mechanisms of injury and disease development," International Immunopharmacology, vol. 2, no. 2-3, pp. 173-182, 2002.

[13] E. Hnizdo and V. Vallyathan, "Chronic obstructive pulmonary disease due to occupational exposure to silica dust: a review of epidemiological and pathological evidence," Occupational and Environmental Medicine, vol. 60, no. 4, pp. 237-243, 2003.

[14] T. Kaewamatawong, A. Shimada, M. Okajima et al., "Acute and subacute pulmonary toxicity of low dose of ultrafine colloidal silica particles in mice after intratracheal instillation," Toxicologic Pathology, vol. 34, no. 7, pp. 958-965, 2006.

[15] E. J. Park and K. Park, “Oxidative stress and pro-inflammatory responses induced by silica nanoparticles in vivo and in vitro," Toxicology Letters, vol. 184, no. 1, pp. 18-25, 2009.

[16] M. Chen and A. von Mikecz, "Formation of nucleoplasmic protein aggregates impairs nuclear function in response to SiO2 nanoparticles," Experimental Cell Research, vol. 305, no. 1, pp. 51-62, 2005.

[17] J. C. K. Lai, G. Ananthakrishnan, S. Jandhyam et al., "Treatment of human astrocytoma U87 cells with silicon dioxide nanoparticles lowers their survival and alters their expression of mitochondrial and cell signaling proteins," International Journal of Nanomedicine, vol. 5, no. 1, pp. 715-723, 2010. 
[18] H. Nabeshi, T. Yoshikawa, K. Matsuyama et al., "Systemic distribution, nuclear entry and cytotoxicity of amorphous nanosilica following topical application," Biomaterials, vol. 32, no. 11, pp. 2713-2724, 2011.

[19] F. A. van Broekhuizen and J. C. van Broekhuizen, "Nanotechnology in the european construction industrystate of the art 2009—executive summary," 2010, http://hesa.etui-rehs.org/uk/newsevents/files/Nano_Executive_ $\% 20$ summary.pdf.

[20] H. Arakawa, K. Honma, Y. Saito et al., "Pleural disease in silicosis: pleural thickening, effusion, and invagination," Radiology, vol. 236, no. 2, pp. 685-693, 2005.

[21] G. Miserocchi, "Mechanisms controlling the volume of pleural fluid and extravascular lung water," European Respiratory Review, vol. 18, no. 114, pp. 244-252, 2009.

[22] C. Boutin, P. Dumortier, F. Rey, J. R. Viallat, and P. De Vuyst, "Black spots concentrate oncogenic asbestos fibers in the parietal pleura: thoracoscopic and mineralogic study," American Journal of Respiratory and Critical Care Medicine, vol. 153, no. 1, pp. 444-449, 1996.

[23] K. M. Muller, I. Schmitz, and K. Konstantinidis, "Black spots of the parietal pleura: morphology and formal pathogenesis," Respiration, vol. 69, no. 3, pp. 261-267, 2002.

\section{This article should be cited as follows:}

Yuguo Song and Shichuan Tang, "Nanoexposure, Unusual Diseases, and New Health and Safety Concerns," TheScientificWorldJOURNAL, vol. 11, pp. 1821-1828, 2011. 

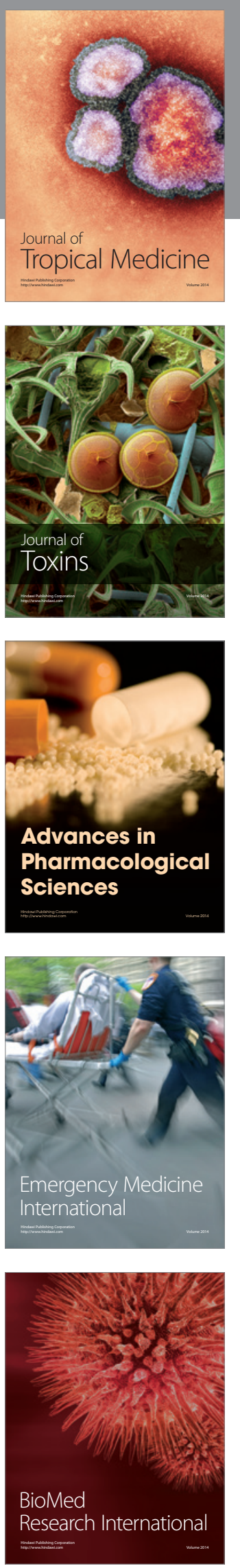
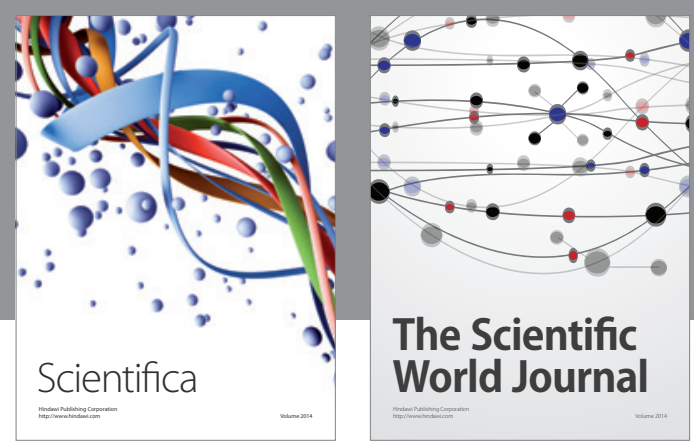

The Scientific World Journal
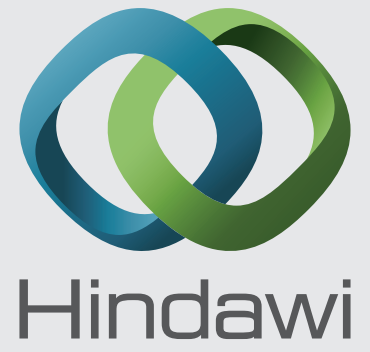

Submit your manuscripts at

http://www.hindawi.com
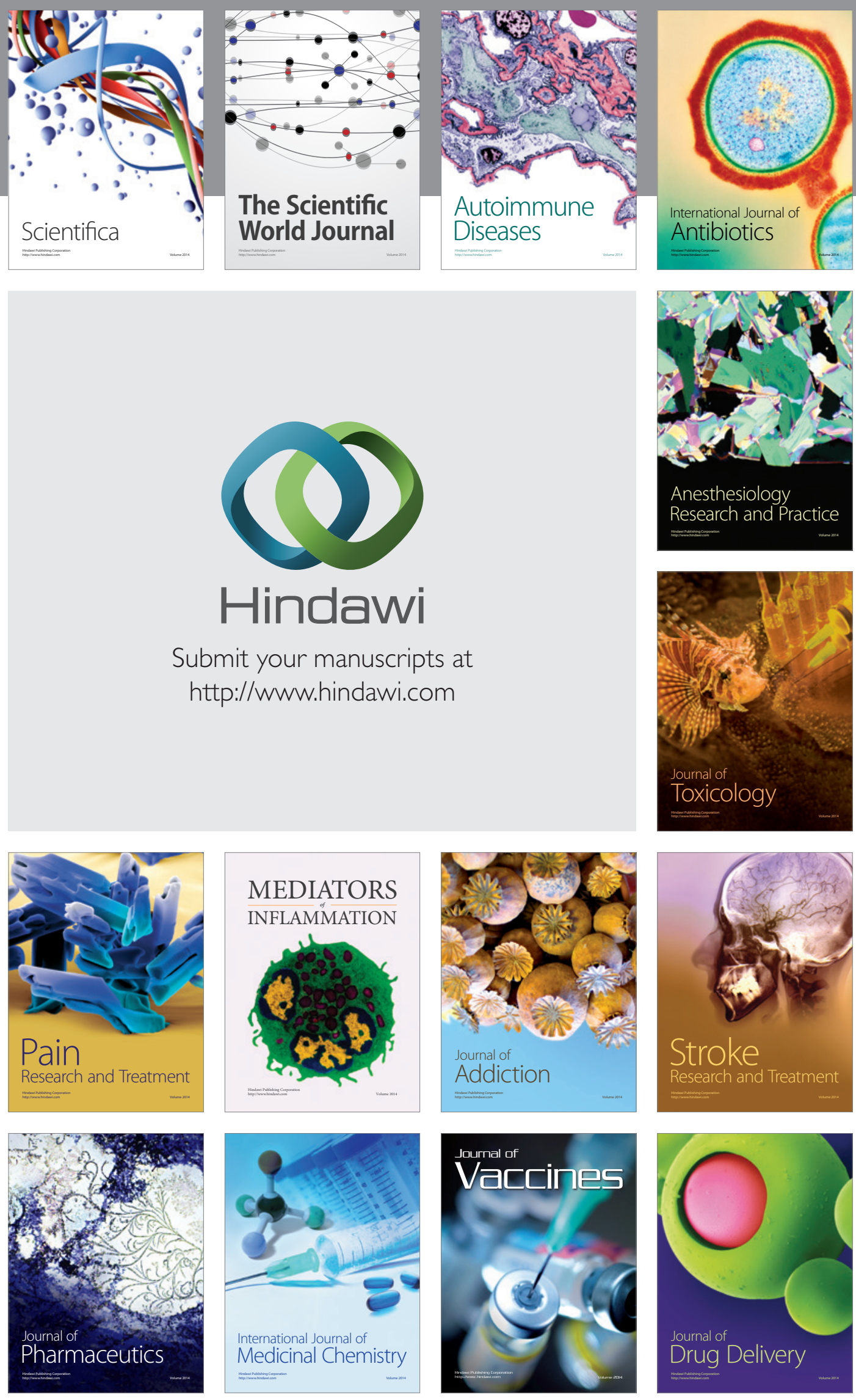